\title{
Heat Capacity Reveals the Physics of a Frustrated Spin Tube
}

\author{
Nedko B. Ivanov, ${ }^{1,2, *}$ Jürgen Schnack, ${ }^{2, \dagger}$ Roman Schnalle, ${ }^{2}$ Johannes Richter, ${ }^{3}$ Paul Kögerler, ${ }^{4}$ Graham N. Newton, ${ }^{5}$ \\ Leroy Cronin, ${ }^{5}$ Yugo Oshima, ${ }^{6}$ and Hiroyuki Nojiri ${ }^{6, \$}$ \\ ${ }^{1}$ Institute of Solid State Physics, Bulgarian Academy of Sciences, Tzarigradsko chaussee 72, 1784 Sofia, Bulgaria \\ ${ }^{2}$ Fakultät für Physik, Universität Bielefeld, Postfach 100131, D-33501 Bielefeld, Germany \\ ${ }^{3}$ Institut für Theoretische Physik, Universität Magdeburg, Post Office Box 4120, D-39016 Magdeburg, Germany \\ ${ }^{4}$ Institut für Anorganische Chemie, RWTH Aachen, Landoltweg 1, D-52074 Aachen, Germany \\ ${ }^{5}$ Department of Chemistry, The University of Glasgow, Glasgow, G12 8QQ, United Kingdom \\ ${ }^{6}$ Institute for Materials Research, Tohoku University, Katahira 2-1-1, Sendai 980-8577, Japan \\ (Received 12 April 2010; revised manuscript received 17 May 2010; published 16 July 2010)
}

\begin{abstract}
We report on theoretical and experimental results concerning the low-temperature specific heat of the frustrated spin-tube material $\left[\left(\mathrm{CuCl}_{2} \mathrm{tachH}\right)_{3} \mathrm{Cl}\right] \mathrm{Cl}_{2}$ (tach denotes 1,3,5-triaminocyclohexane). This substance turns out to be an unusually perfect spin-tube system which allows to study the physics of quasione-dimensional antiferromagnetic structures in rather general terms. An analysis of the specific-heat data demonstrates that at low enough temperatures the system exhibits a Tomonaga-Luttinger liquid behavior corresponding to an effective spin-3/2 antiferromagnetic Heisenberg chain with short-range exchange interactions. On the other hand, around $2 \mathrm{~K}$ the composite spin structure of the chain is revealed through a Schottky-type peak in the specific heat. We argue that the dominating contribution to the peak originates from gapped magnon-type excitations related to the internal degrees of freedom of the rung spins.
\end{abstract}

DOI: 10.1103/PhysRevLett.105.037206

PACS numbers: 75.10.Jm, 75.40.Mg, 75.50.Ee, 75.50.Xx

Introduction.-Spin tubes constitute a special class of quasi-one-dimensional spin ladder systems characterized by periodic boundary conditions in the rung direction [113]. The magnetic compound $\left[\left(\mathrm{CuCl}_{2} \mathrm{tachH}\right)_{3} \mathrm{Cl}\right] \mathrm{Cl}_{2}$, for sample preparation, see [14], is a geometrically frustrated triangular spin tube, the frustration being related both to the triangular arrangement of its rungs and to the twisted geometry of the legs, compare Fig. 1(a). The relatively simple exchange pathway structure, that thanks to high symmetry is described by only two dominant Heisenberg exchange couplings [15], as well as the extremely weak exchange interactions between neighboring tubes, renders $\left[\left(\mathrm{CuCl}_{2} \mathrm{tachH}\right)_{3} \mathrm{Cl}\right] \mathrm{Cl}_{2}$ an excellent real material to study general properties of the spin-tube systems [4,6,9,11]. An appropriate spin Hamiltonian describing the magnetic properties of this material reads as

$$
\begin{aligned}
\mathcal{H}= & \sum_{n=1}^{L} \sum_{\alpha=1}^{3}\left[J_{1} \boldsymbol{\sigma}_{n, \alpha} \cdot \boldsymbol{\sigma}_{n, \alpha+1}\right. \\
& \left.+J_{2} \boldsymbol{\sigma}_{n, \alpha} \cdot\left(\boldsymbol{\sigma}_{n+1, \alpha+1}+\boldsymbol{\sigma}_{n+1, \alpha-1}\right)\right],
\end{aligned}
$$

where $\boldsymbol{\sigma}_{n, \alpha}(\alpha=1,2,3)$ are spin- $1 / 2$ operators defined on the vertices of the elementary triangle denoted by index $n$ $(n=1, \ldots, L)$. As depicted in Fig. 1(b), the twisted spin tube may also be thought of as a three-leg ladder with periodic boundary conditions in the rung direction, where the parameters $J_{1}$ and $J_{2}$ are strengths of the rung and crossing (diagonal) exchange bonds, respectively. $\left[\left(\mathrm{CuCl}_{2} \mathrm{tachH}\right)_{3} \mathrm{Cl}\right] \mathrm{Cl}_{2}$ is characterized by the parameters $J_{1} / k_{B}=1.8 \mathrm{~K}$ and $J_{2} / k_{B}=3.9 \mathrm{~K}$ [15], whereas the leg exchange constant $J_{2}^{\prime}>0$, introduced for the sake of clarity in Fig. 1(b), seems to vanish in this material. Figure 1(b) clearly reveals the translation symmetry with one triangle per unit cell.

Two extreme scenarios for the ground state of Eq. (1) with antiferromagnetic couplings $\left(J_{1}, J_{2}>0\right)$ were outlined in Ref. [9]. In the case of dominating $J_{1}$ couplings, the system maps onto an effective spin-chirality model, where the additional chirality degrees of freedom appear as a result of the ground-state degeneracy of each individual triangle. On the other hand, for dominating $J_{2}$ couplings the system maps onto an effective spin-3/2 antiferromag-

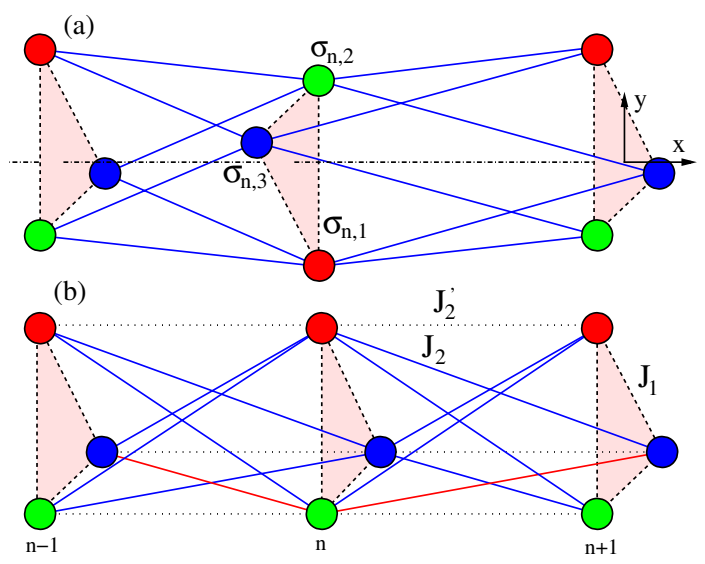

FIG. 1 (color online). (a) Sketch of the twisted spin-tube system. (b) An equivalent spin model obtained through inversion of every second triangle. The $n$th elementary cell contains the spin- $1 / 2$ operators $\boldsymbol{\sigma}_{n, 1}, \boldsymbol{\sigma}_{n, 2}$, and $\boldsymbol{\sigma}_{n, 3}$. 
netic Heisenberg chain (AHC) containing additional biquadratic exchange couplings.

In this Letter, we demonstrate by means of specific-heat measurements, that the low-temperature properties of the spin-tube material $\left[\left(\mathrm{CuCl}_{2} \mathrm{tachH}\right)_{3} \mathrm{Cl}\right] \mathrm{Cl}_{2}$ reproduce the behavior of a spin-3/2 AHC characterized by the effective short-range exchange-coupling constant $J_{\text {eff }}=2 J_{2} / 3$. Since the exchange interactions between different tubes are extremely small, the discussed compound also provides a rare example of spin-3/2 AHC [16-20]. The experimental observables strongly suggest that if the system ever orders this should be much below $0.1 \mathrm{~K}$. At elevated temperatures the measured specific heat exhibits a big Schottky-type peak located around $T \approx 2 \mathrm{~K}$. A detailed analysis-combining the semiclassical spin-wave approach with a number of numerical techniques such as the quantum Monte Carlo (QMC) method, the Lanczos exact numerical diagonalization (ED), and the complete exact diagonalization [21,22]—implies that the main contribution to the specific-heat peak stems from the lowestlying gapped magnon excitations resulting from the internal degrees of freedom of the composite rung spins.

Theoretical background.-Before discussing the experimental results it is instructive to address the structure of the low-energy part of the spectrum of Hamiltonian (1). To this end we rewrite Eq. (1) in the form

$$
\mathcal{H}=\sum_{n=1}^{L}\left[\frac{J_{1}}{2}\left(\mathbf{s}_{n}^{2}-\frac{9}{4}\right)+J_{2} \boldsymbol{s}_{n} \cdot \boldsymbol{s}_{n+1}\right]+V .
$$

Here $\boldsymbol{s}_{n}=\boldsymbol{\sigma}_{n, 1}+\boldsymbol{\sigma}_{n, 2}+\boldsymbol{\sigma}_{n, 3}$ is the rung spin operator related to the $n$th triangle. The number of $\mathrm{Cu}$ sites is $N=$ $3 L$, where $L$ is the number of rungs. The interaction term $V$ reads as

$$
V=\left(J_{2}^{\prime}-J_{2}\right) \sum_{n=1}^{L} \sum_{\alpha=1}^{3} \boldsymbol{\sigma}_{n, \alpha} \cdot \boldsymbol{\sigma}_{n+1 \alpha},
$$

where we have included an additional leg exchange interaction $J_{2}^{\prime}$, see Fig. 1(b). The last two equations explicitly show that the condition $J_{2}=J_{2}^{\prime}$ defines a special case where the model for $s_{n}=3 / 2$ maps on an effective spin-3/2 AHC. Up to first order in $J_{2}^{\prime}-J_{2}$, the interaction term $V$ in Eq. (2) results in an effective exchange constant for this effective spin-3/2 AHC: $J_{\text {eff }}=J_{2}+\left(J_{2}^{\prime}-J_{2}\right) / 3$. At the special point $J_{2}^{\prime}=0$, the result $J_{\text {eff }}=2 J_{2} / 3$ coincides with the first-order result of Ref. [9], obtained by another perturbation scheme.

The above considerations imply that a qualitative picture of the low-lying spin excitations can be obtained already in the framework of the semiclassical spin-wave approach starting from the classical Néel configuration $\left|S_{t},-S_{t}, \ldots\right\rangle$, where $S_{t}$ is the maximal value of the $z$ component of the rung spin $\left(S_{t}=3 / 2\right.$ in the present case). Since the elementary cell contains three spin- $S$ variables, there appear three different branches of spinwave modes

$$
\begin{aligned}
E_{m}\left(k_{x}\right)= & 4 S J_{2} \\
& \times \sqrt{\left(1-\alpha \sin ^{2} \frac{k_{y}}{2}\right)^{2}-\frac{1}{4}\left[\cos k_{x}+\cos \left(k_{x}+k_{y}\right)\right]^{2}},
\end{aligned}
$$

where $\alpha=J_{1} / J_{2}, k_{y}=2 \pi m / 3(m=0,1,2)$, and $S$ is the spin of a single site ( $S=1 / 2$ in the present case). As may be expected, the energy of the $m=0$ branch does not depend on the parameter $J_{1}$, since it is related to the dynamics of the cell spins $\boldsymbol{s}_{n}$ as a whole

$$
E_{0}\left(k_{x}\right)=v_{s}\left|\sin k_{x}\right|, \quad v_{s}=(6 S) \frac{2 J_{2}}{3} .
$$

The above expressions reproduce the well-known semiclassical results for the dispersion relation and the related spin-wave velocity $v_{s}$ of a spin- $(3 \cdot S)$ AHC with the effective exchange constant $J_{\text {eff }}=2 J_{2} / 3$.

The dispersion relations of the $k_{y}=2 \pi / 3$ and $k_{y}=$ $4 \pi / 3$ excitations can be expressed in the following form

$$
E_{1,2}\left(k_{x}\right)=\sqrt{\Delta^{2}+4 S^{2} J_{2}^{2} \sin ^{2}\left(k_{x} \mp \frac{2 \pi}{3}\right)},
$$

where the excitation gap $\Delta=4 S J_{2} \sqrt{(1-3 \alpha / 4)^{2}-1 / 4}$ corresponds to the lowest-lying modes at wave vectors $k_{x}=\pi / 3$ and $2 \pi / 3$.

The solid curves in Fig. 2 show the dispersion relations of the discussed spin-wave excitations. On the other hand, the symbols depict the positions of the lowest-lying triplet states, as obtained by the ED method for periodic clusters containing $L=8$ and 12 unit cells. Apart from the finitesize effects related to the ED results, it is clearly seen that both methods qualitatively yield similar results. As a matter of fact, the discussed spin-wave branches may also be considered as one-dimensional analogs of the three spinwave branches in the triangular lattice antiferromagnet. In this respect, the lowest-lying excitations at $k_{x}=0,2 \pi / 3$

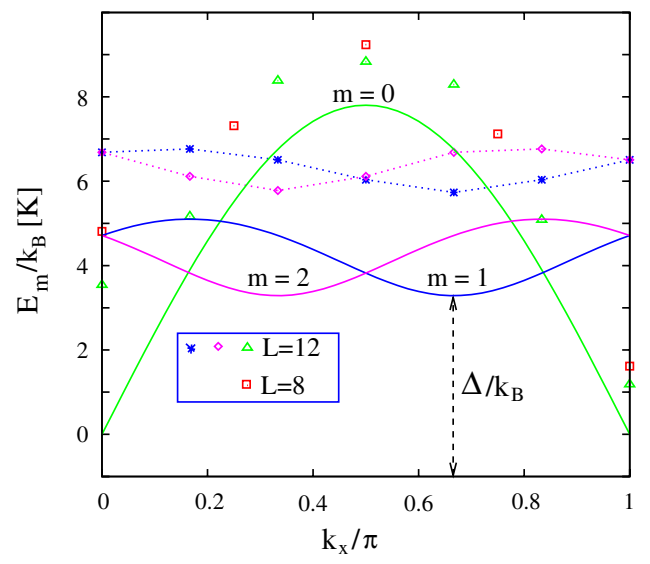

FIG. 2 (color online). Spin-wave excitation modes [solid curves, Eqs. (5) and (6)] compared with the lowest triplet excitations in two periodic clusters (symbols). 
and $4 \pi / 3$ in the spin tube are one-dimensional analogs of the three Goldstone modes in the triangular lattice antiferromagnet.

More accurate estimates for the parameters of the excitation spectrum $v_{s}$ and $\Delta$ can be obtained from an extrapolation of the ED results for $L=6,8,10$, and 12 unit cells. Using the approach of Ref. [17], one finds the following estimates from the extrapolations of $E_{0}(2 \pi / L)$ vs $\sin (2 \pi / L) / L$ and $E_{1}(2 \pi / 3)$ vs $1 / L$ (see Fig. 3 ):

$$
v_{s}=10.06 \mathrm{~K}=3.87 \frac{2 J_{2}}{3 k_{B}}, \quad \Delta / k_{B}=5.32 \mathrm{~K} .
$$

Interestingly, the extrapolation result $3 v_{s} /\left(2 J_{2} / k_{B}\right)=3.87$ exactly reproduces the density-matrix renormalization group estimate for the spin-wave velocity of the spin-3/2 AHC characterized by the exchange constant $2 J_{2} / 3$ [17]. As already discussed, the same effective exchange constant $\left(J_{\text {eff }}=2 J_{2} / 3\right)$ arises both in the first-order result for the effective spin model and in the semiclassical spin-wave approach. Below we demonstrate numerically that the specific heat of a spin-3/2 AHC with the exchange constant $2 J_{2} / 3$ excellently reproduces the experimental results in the low-temperature region $T \leq 0.5 \mathrm{~K}$.

Experiment.-The specific heat of polycrystalline samples of $100 \mu \mathrm{g}$ of $\left[\left(\mathrm{CuCl}_{2} \mathrm{tachH}\right)_{3} \mathrm{Cl}\right] \mathrm{Cl}_{2}$, symbols in Figs. 4, was measured by the relaxation method down to $120 \mathrm{mK}$ by using a microcalorimeter. Technical details are given in Ref. [23]. To obtain the magnetic specific heat, (i) the heat capacity of the holder and stage and (ii) the contribution of phonons are subtracted. The former is done by using the blank data without sample. The latter is approximated by a Debye-like specific-heat contribution, where the prefactor of $T^{3}$ is determined so that the magnetic entropy saturates around $10 \mathrm{~K}$. Thanks to the small exchange couplings the important features of the magnetic specific heat are practically not altered by phonons below $T \approx 3 \mathrm{~K}$ (phonon contribution less than $1 \%$ at $2 \mathrm{~K}$ and about $7 \%$ at $4 \mathrm{~K}$ ). A more detailed description of the experimental result will be published separately.

Analysis of the experimental results.-The solid curve in Fig. 4 depicts the QMC result for the specific heat of the spin-3/2 periodic AHC composed of 100 spin sites, that is evaluated employing the ALPS code [24]. As an effective
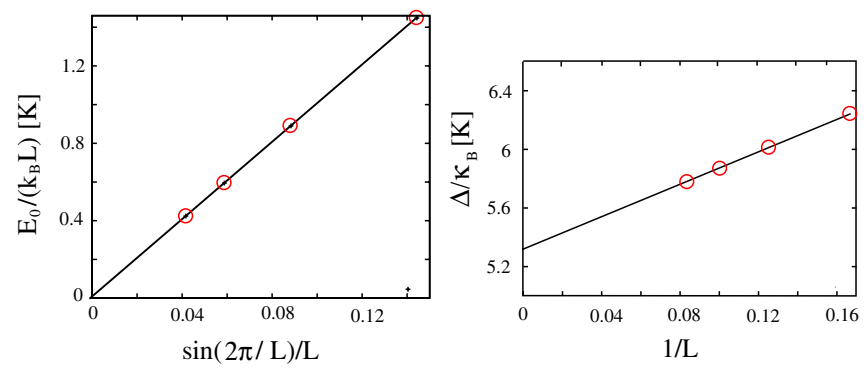

FIG. 3 (color online). Extrapolation of the ED results giving the parameters of the excitation spectrum $v_{s}$ (left) and $\Delta$ (right). exchange parameter we used $J_{\text {eff }}=2 J_{2} / 3$. As can be seen in Fig. 4, the QMC result reproduces the experimental data very well in the region $T \leq 0.5$. This means that even for $J_{2}^{\prime}=0$, when the parameter $\left(J_{2}^{\prime}-J_{2}\right)$ is definitely not small, the low-energy physics of Hamiltonian (1) is described by the spin-3/2 AHC. Therefore, it turns out that the theoretically predicted biquadratic exchange term [3,9] plays no role in the experimentally interesting region of the phase diagram characterized by the dimensionless parameter $J_{1} / J_{2}=0.46$.

As generally accepted, the spin-3/2 AHC and the spin-1/2 AHC belong to the same universality class characterized by the conformal central charge $c=1$ [17]. Therefore, in the extremely low-temperature regime we expect the universal relation

$$
\frac{C(T)}{N k_{B}}=\frac{\pi c T}{9 v_{s}}
$$

with the velocity of the gapless spin excitations $v_{s}=$ $10.06 \mathrm{~K}$. As clearly seen in Fig. 4, already for $T \leq 0.5 \mathrm{~K}$ the measured specific heat not only coincides with the QMC result, but also nicely extrapolates towards the universal behavior represented by Eq. (8). The latter observations not only strongly imply that the spin-tube compound $\left[\left(\mathrm{CuCl}_{2} \mathrm{tachH}\right)_{3} \mathrm{Cl}\right] \mathrm{Cl}_{2}$ is characterized by a gapless Tomonaga-Luttinger liquid ground state, they also rule out the other possibility of $c=9 / 5$ related to the universality class of spin- $S$ integrable models [17]. In a recent report, nuclear magnetic resonance measurements also indicate a gapless spin state in the same material based

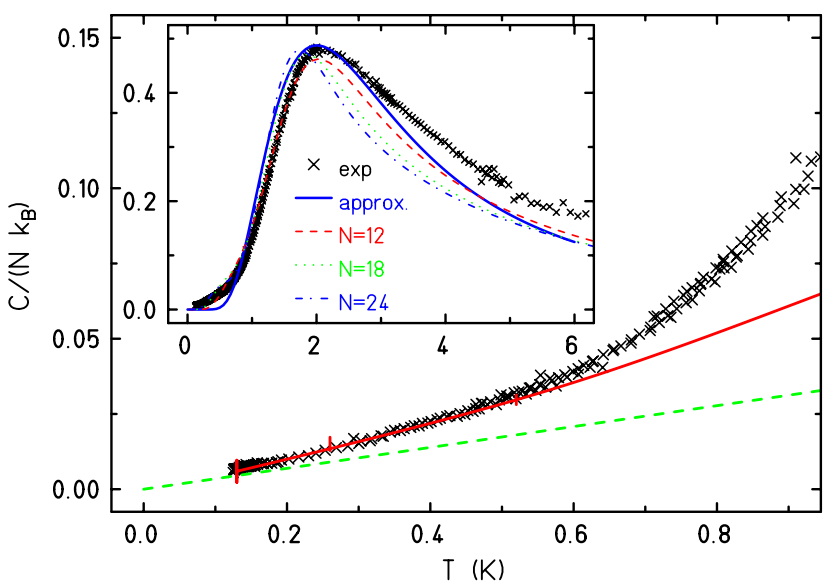

FIG. 4 (color online). Specific heat (per $\mathrm{Cu}$ spin) of $\left[\left(\mathrm{CuCl}_{2} \mathrm{tachH}\right)_{3} \mathrm{Cl}\right] \mathrm{Cl}_{2}$. The symbols always denote the experimental values. Main figure: The solid curve is the QMC result for a spin-3/2 chain of length $L=100$. The dashed line provides the linear specific heat corresponding to the universal Tomonaga-Luttinger liquid form presented by Eq. (8), by using the extrapolation result $v_{s}=10.06 \mathrm{~K}$. Inset: The solid curve depicts the two-level approximation (9). The broken curves denote the specific heat for three complete diagonalizations for finite sizes. 
on estimates for the extremely low-temperature part of the magnetic susceptibility [25].

To explain the experimental data at intermediate temperatures around $2 \mathrm{~K}$, we use the established structure of the low-lying excitation spectrum, compare Fig. 2. As a rough approximation, one may take $E_{1,2}\left(k_{x}\right) \approx \Delta$ and use the well-known expression for the specific heat of a twolevel system (with the assumption that the excited level has twice the weight of the ground state, i.e., $r=2$ in the following expression). Since the exact density of states is not known in the thermodynamic limit, we use an overall parameter $A$ in order to fix the height of the Schottky peak:

$$
\frac{C}{N k_{B}}=A \frac{r(\Delta / T)^{2} \exp (\Delta / T)}{[\exp (\Delta / T)+r]^{2}} .
$$

Notice that the position of the peak does not depend on the value of $A$. The expression for $C(T)$, Eq. (9), with $\Delta / k_{B}=$ $5.32 \mathrm{~K}$ is plotted in the inset of Fig. 4 by a thick curve together with the experimental data. One observes that it reproduces very well not only the position of the peak $\left(T_{m} \approx 2 \mathrm{~K}\right)$ but also the behavior of the specific heat for $T<T_{m}$ down to $T \approx 0.7 \mathrm{~K}$, where the contribution from the gapless branch $E_{0}\left(k_{x}\right)$ becomes important. In addition, in the inset of Fig. 4 we also show the specific heat that results from complete diagonalizations of a few finite-size periodic clusters. As easily seen, the overall agreement is good, in spite of the pronounced finite-size effects. This agreement also demonstrates the consistency with previous magnetization data, from which the exchange couplings had been derived [15]. Concerning the observed discrepancies for higher temperatures $(T \geqslant 3 \mathrm{~K}$ ), they could possibly be related to finer details of the phonon spectral density as suggested for other quasi one-dimensional materials [26].

Summary.-We demonstrated that the low-temperature specific-heat behavior of the spin-tube compound $\left[\left(\mathrm{CuCl}_{2} \mathrm{tachH}\right)_{3} \mathrm{Cl}\right] \mathrm{Cl}_{2}$ suggests a Tomonaga-Luttinger liquid type ground state for this material, corresponding to an effective spin-3/2 antiferromagnetic Heisenberg chain characterized by the short-ranged exchange-coupling constant $J_{\text {eff }}=2 J_{2} / 3$. On the other hand, we argued that the main contribution to the observed Schottky-type peak around $T \approx 2 \mathrm{~K}$ comes from the lowest-lying gapped magnon-type excitations resulting from the internal degrees of freedom of the composite rung spins.

Computing time at the Leibniz Computing Center in Garching is gratefully acknowledged. We also thank Andreas Honecker and David Johnston for fruitful discussions as well as Tao Xiang for explaining his transfer matrix results to us. J.S. is grateful to Andreas Läuchli for advising how to run the ALPS code [24]. This work was supported by the DFG (FOR 945, SCHN 615/13-1) and the Bulgarian Science Foundation under the Grant No. DO02-
264. H. N. is supported by Kakenhi No. 20244052 from JSPS.

*Nedko.Ivanov@Physik.Uni-Magdeburg.DE †jschnack@uni-bielefeld.de nojiri@imr.tohoku.ac.jp

[1] P. Millet, J. Y. Henry, F. Mila, and J. Galy, J. Solid State Chem. 147, 676 (1999).

[2] J. L. Gavilano, D. Rau, S. Mushkolaj, H. R. Ott, P. Millet, and F. Mila, Phys. Rev. Lett. 90, 167202 (2003).

[3] A. Lüscher, R. M. Noack, G. Misguich, V. N. Kotov, and F. Mila, Phys. Rev. B 70, 060405(R) (2004).

[4] M. Sato, Phys. Rev. B 72, 104438 (2005).

[5] M. Matsumoto, T. Sakai, M. Sato, H. Takayama, and S. Todo, Physica (Amsterdam) 29E, 660 (2005).

[6] T. Sakai, M. Matsumoto, K. Okunishi, K. Okamoto, and M. Sato, Physica (Amsterdam) 29E, 633 (2005).

[7] T. Saha-Dasgupta, R. Valentí, F. Capraro, and C. Gros, Phys. Rev. Lett. 95, 107201 (2005).

[8] K. Okunishi, S. Yoshikawa, T. Sakai, and S. Miyashita, Prog. Theor. Phys. Suppl. 159, 297 (2005).

[9] J.-B. Fouet, A. Läuchli, S. Pilgram, R. M. Noack, and F. Mila, Phys. Rev. B 73, 014409 (2006).

[10] M. Sato and T. Sakai, Phys. Rev. B 75, 014411 (2007).

[11] S. Nishimoto and M. Arikawa, Phys. Rev. B 78, 054421 (2008).

[12] O. Zaharko et al., Phys. Rev. B 78, 214426 (2008).

[13] M. Sato, Phys. Rev. B 75, 174407 (2007).

[14] G. Seeber, P. Kögerler, B. M. Kariuki, and L. Cronin, Chem. Commun. (Cambridge) 1580 (2004).

[15] J. Schnack, H. Nojiri, P. Kögerler, G. J. T. Cooper, and L. Cronin, Phys. Rev. B 70, 174420 (2004).

[16] T. Xiang, Phys. Rev. B 58, 9142 (1998).

[17] K. Hallberg, X. Q. G. Wang, P. Horsch, and A. Moreo, Phys. Rev. Lett. 76, 4955 (1996).

[18] A. Klümper and D. C. Johnston, Phys. Rev. Lett. 84, 4701 (2000).

[19] D. C. Johnston, R. K. Kremer, M. Troyer, X. Wang, A. Klümper, S. L. Bud'ko, A. F. Panchula, and P. C. Canfield, Phys. Rev. B 61, 9558 (2000).

[20] S. Itoh, Y. Endoh, K. Kakurai, H. Tanaka, S. M. Bennington, T. G. Perring, K. Ohoyama, M. J. Harris, K. Nakajima, and C. D. Frost, Phys. Rev. B 59, 14406 (1999).

[21] J. Schnack, P. Hage, and H.-J. Schmidt, J. Comput. Phys. 227, 4512 (2008).

[22] R. Schnalle and J. Schnack, Phys. Rev. B 79, 104419 (2009).

[23] T. Zenmoto, Y. Oshima, H. Nojiri, S. H. Lee, and M. Kofu, J. Low Temp. Phys. 159, 118 (2010).

[24] A. Albuquerque et al., (ALPS Collaboration), J. Magn. Magn. Mater. 310, 1187 (2007).

[25] Y. Furukawa, Y. Sumida, K. Kumagai, H. Nojiri, P. Kögerler, and L. Cronin, J. Phys. Conf. Ser. 150, 042036 (2009).

[26] J. Hone, B. Batlogg, Z. Benes, A. Johnson, and J. Fischer, Science 289, 1730 (2000). 